\title{
Hausarztmangel: Kassen bremsen die Hausarztmedizin
}

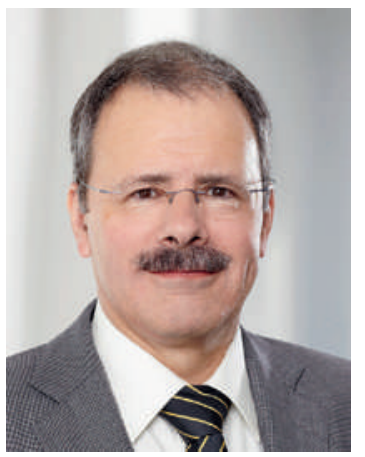

Voraussichtlich im kommenden Frühling entscheidet das Parlament über die Parlamentarische Initiative von Nationalrat Olivier Feller «Keine Benachteiligung von Fachärztinnen und Fachärzten für Allgemeine Innere Medizin mit einem zweiten Facharzttitel». Die FMH unterstützt diesen Vorstoss ganz klar. Würde das Krankenversicherungsgesetz entsprechend angepasst, wären ungerechtfertigte Ausschlüsse von Doppeltitelträgern nicht mehr möglich.

Das Problem schaffen gewisse Krankenversicherer, die nicht bereit sind, Fachärzte für Allgemeine Innere Medizin in ihre einseitig definierten Listen der ärztlichen Grundversorgerinnen und Grundversorger aufzunehmen, wenn diese einen zweiten Fachtitel erworben haben (beispielsweise in Allergologie, Immunologie, Rheumatologie oder Endokrinologie).

Hinter dieser Haltung einzelner Kassen steht offenbar der Verdacht, ein Hausarzt mit einem weiteren Facharzttitel behandle seine Hausarzt-Patienten teurer als der Kollege, der ausschliesslich Grundversorger ist. Diese Annahme müsste belegt werden. Sie ist es nicht.

\section{Schliessen Krankenversicherungen Doppeltitelträger mit einem Facharzt in Allgemeiner Innerer Medizin aus, verstossen sie gegen den Grundsatz der Gleichbehandlung.}

Konkret bedeutet der Entscheid einer Kasse, Doppeltitelträger als Grundversorger auszuschliessen, dass deren Patientinnen und Patienten von Kassen-definierten Listenmodellen ausgeschlossen sind. Entscheiden sich diese Patienten trotzdem für ein solches Modell - was gerade in diesen Wochen, in denen viele Leute in ein Hausarzt-Listenmodell wechseln möchten, oft der Fall sein dürfte! -, dann müssen sie ihren Hausarzt wechseln.

Das ist absurd: Die Kenntnis der Krankengeschichte und das Vertrauensverhältnis müssen durch Konsultationen beim neuen Hausarzt neu aufgebaut werden. Die Frage der FMH sei erlaubt, wie viel solch erzwungene Wechsel unter dem Strich kosten.

Die Haltung der Versicherer ist auch gesundheitspolitisch verkehrt. Vergessen wir nicht: Wir haben in der Schweiz einen Mangel an Hausärzten! Statt den Zugang zur Hausarztmedizin zu behindern, sollten Kassen alles daran setzen, Grundversorgungsmodelle und ärztliche Qualität zu erhalten

\section{Müssen Patienten ihren Hausarzt wechseln, geht viel Wissen verloren, was zu Mehrkosten führen kann.}

und zu fördern. Hausärzte mit einem zweiten Facharzttitel haben viel Wissen und breite Erfahrung. Sie können Zweitkonsultationen verhindern und entsprechende Mehrkosten verringern. Kommt dazu: Wenn Doppeltitelträger als Grundversorger aus den Hausarzt-Listenmodellen ausgeschlossen werden, riskiert man, sie als Notfallärzte zu verlieren. Können wir uns das leisten?

Aus Sicht der FMH ist der Ausschluss von Doppeltitelträgern auch juristisch fragwürdig. Er ist willkürlich und verstösst gegen den Grundsatz der Gleichbehandlung. Die FMH lehnt einseitig kassendeklarierte Listenmodelle ab und empfiehlt partnerschaftliche Modelle.

Bisher bin ich immer davon ausgegangen, dass Wissen und Bildung wünschenswert und $\mathrm{zu}$ fördern sind. Unter anderem deshalb, weil sie zentrale Faktoren sind, welche Menschen persönlich wachsen lassen und nachhaltig zur Entwicklung eines Landes beitragen. Aber anscheinend müssen wir Ärztinnen und Ärzte ganz sorgfältig darauf achten, ja nicht zu viel zu wissen, sonst werden wir noch dafür bestraft ...

Dr. med. Jürg Schlup, Präsident der FMH 